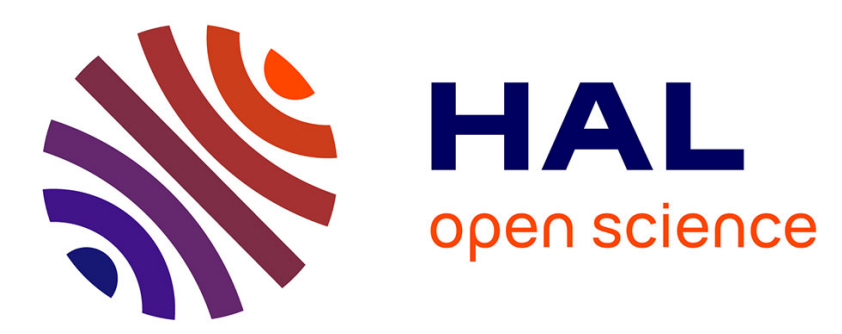

\title{
A population balance model for the prediction of breakage of emulsion droplets in SMX+ static mixers
}

\author{
Noureddine Lebaz, Nida Sheibat-Othman
}

\section{To cite this version:}

Noureddine Lebaz, Nida Sheibat-Othman. A population balance model for the prediction of breakage of emulsion droplets in SMX+ static mixers. Chemical Engineering Journal, 2019, 361, pp.625-634. 10.1016/j.cej.2018.12.090 . hal-01966545

\section{HAL Id: hal-01966545 \\ https://hal.science/hal-01966545}

Submitted on 18 Nov 2020

HAL is a multi-disciplinary open access archive for the deposit and dissemination of scientific research documents, whether they are published or not. The documents may come from teaching and research institutions in France or abroad, or from public or private research centers.
L'archive ouverte pluridisciplinaire HAL, est destinée au dépôt et à la diffusion de documents scientifiques de niveau recherche, publiés ou non, émanant des établissements d'enseignement et de recherche français ou étrangers, des laboratoires publics ou privés. 


\title{
A population balance model for the prediction of breakage of emulsion droplets in SMX+ static mixers
}

\author{
Noureddine Lebaz ${ }^{\mathrm{a}}$, Nida Sheibat-Othman ${ }^{\mathrm{a}, *}$ \\ ${ }^{a}$ Univ Lyon, Université Claude Bernard Lyon 1, CNRS, LAGEP UMR 5007, 43 boulevard du 11 novembre 1918, F-69100, \\ Villeurbanne, France
}

\begin{abstract}
This work deals with the numerical and experimental investigation of droplets breakage in SMX+ static mixers. While steady-state empirical correlations are commonly used for the prediction of the mean droplets size (e.g. Sauter mean diameter) during emulsification in static mixers, in the present study, a population balance equation (PBE) -based model is developed for the prediction of the dynamic evolution of the droplet size distribution (DSD). The system silicon oil-in-water stabilized by Polysorbate 20 (Tween20 ${ }^{\circledR}$ ) is considered under dilute conditions $(<5 \%$ vol. $)$. Due to the physico-chemical properties of the system and the operating conditions, the droplets breakage process is dominant, while coalescence and Ostwald ripening are negligible. The breakage kernel proposed by Alopaeus et al. [1] is employed and its parameters are identified and validated for the present system under different operating conditions. The effects of the number of SMX+ elements, viscosity and fraction of the dispersed phase, the DSD at the inlet of the mixers as well as the volume-average energy dissipation rate within the SMX+ elements on the evolution of the DSD are investigated. The model was found to be able of predicting the DSD over a wide range of operating conditions.
\end{abstract}

Keywords: Population balance, static mixers, breakage, droplet size distribution, liquid-liquid dispersion

\footnotetext{
${ }^{*}$ Corresponding author. Address: LAGEP Université Claude Bernard Lyon 1, bt 308G ESCPE-Lyon, 43 bd du 11 Novembre 1918, Villeurbanne 69622 France. Tel.: +330472 4318 50; Fax: +3304 72431699

Email address: nida.othman@univ-lyon1.fr (Nida Sheibat-Othman)
} 


\section{Introduction}

Static mixers, also called motionless or in-line mixers, are employed in many industrial fields as mixing devices, as mass transfer enhancers between phases and/or heat exchangers since they achieve high mixing performances in short residence times [2]. This alternative technology to mechanical stirred vessels represents a suitable option for process intensification due to its compactness and continuous operating mode, and offers several economical advantages mainly due to lower energy consumption and maintenance costs [3, 4, 5].

Static mixers consist of non-moving complex porous structures inserted into cylindrical pipes and aligned in a particular arrangement [6]. Various designs of static mixers are commercially available, the most used being the Kinecs ${ }^{\circledR}$, Ross Low-Pressure Drop and Low-Low-Pressure Drop and the Sulzer SMV ${ }^{\circledR}$ and $\mathrm{SMX}^{\circledR}$ 7]. Because of the porous structure of the mixers, the fluid flow is constantly spatially redirected which minimizes dead zones and leads to a very efficient mixing in the radial direction but causes pressure drop as well [8]. They can therefore ensure good mixing as well as dispersion of multiphase systems, such as liquid/liquid emulsification. In emulsification process, mixing is a decisive operation as it directly controls the droplet size distribution (DSD) which is a determinant product quality affecting its rheological properties and stability, besides control of mass transfer between phases in some applications [9]. The use of static mixers in emulsification processes offers the advantage of uniform hydrodynamic conditions, since the spatial variation of the local energy dissipation rate is low with a short residence time, which allows a better control of the DSD [10]. Moreover, a quite intense average energy dissipation is available in static mixers (of the order of few hundreds of Watts per kg) compared to stirred tank reactors (at the best few Watts per kg), that can easily be manipulated by modifying the operating conditions.

The dispersion efficiency in static mixers is a complex process depending on hydrodynamics phenomena, turbulence structure and physicochemical properties of the multiphase system [11]. Steady-state correlations are commonly used to predict the mean droplet diameter in these systems. These correlations are generally 
derived from models developed for batch stirred tanks and relate the droplets' Sauter mean diameter to the Reynolds, Newton and Weber adimensional numbers and some of them take into account the viscosity ratio or the dispersed phase fraction [6, 12, 13, 14, 15, 16, 17. However, these correlations assume hydrodynamic equilibrium, which means that the droplet size of the emulsion at the output of the mixers is independent of the injected size, which is usually not valid. Most of these correlations also assume that the number of mixers is sufficient enough to ensure steady state, and do not give the possibility to predict the droplet size with a lower number of mixers, except few of them. Moreover, these correlations are semi-empirical, which limits the range of their validity to specific conditions under which they were identified. Finally, no information on the polydispersity of the DSD is reachable with this approach since they give only the mean droplet size.

Fundamental population balance equation-based models have extensively been investigated in recent years to predict the evolution of the DSD in well mixed liquid-liquid systems [18, 19, 20, 21, 22, 23]. These models were recently applied to systems with non-uniform spatial energy and turbulence dissipation, such as high pressure homogenizers 24, 9, 25, 26. In this context, multi-scale modeling has also been considered in such non-uniform systems, by coupling computational fluid dynamic (CFD) simulations with the PBE model in order to investigate local droplet breakage and coalescence [27, 28, 29, 30. However, in static mixers, only few such studies can be reported. Azizi \& Taweel [31] developed a PBE model accounting for droplet breakage and coalescence taking place in turbulently flowing dilute oil-in-water emulsions ( $0.5 \%$ oil volume fraction) using multi-stage screen-type static mixers. They adopted the phenomenological model of Coulaloglou \& Tavlarides [32] for both breakage and coalescence. The spatial variation of the energy dissipation rate was adopted from the decay of grid-generated turbulence described by a power profile and included in the PBE model [33. Jaworski et al. 34] studied turbulent flow and droplet breakage in a Kenics static mixer by coupling CFD simulations with a PBE model. The quadrature method of moments was used to solve the transport equations for the moments of the DSD. As the oil volume fraction was very low $(0.1 \%)$, the 
breakage process was dominant and a power law model was chosen for its frequency [35]. CFD simulations were carried out using a two-phase Eulerian approach based on the solution of the Reynolds-averaged NavierStokes equations with the standard $k-\varepsilon$ model for the Reynolds stresses. Using 24 standard Kenics inserts, the effect of the geometry mesh and the superficial velocity on the final DSD were numerically investigated and the results were compared to those obtained experimentally by Berkman \& Calabrese [36]. The deviation between the simulated and the experimental Sauter mean diameter was around $10 \%$ for the best case. Bayraktar et al. 37. presented a numerical methodology for one-way coupling of CFD and PBE and applied it in the case of oil-in-water emulsification using SMV static mixers, but only one experiment was used to demonstrate the potential of this approach. More recently, Farzi et al. 38] developed a two-phase CFD model to assess the emulsification efficiency in a continuous Kenics static mixer. The high-order least-squares method was used to solve the PBE accounting for breakage and coalescence processes [39]. CFD simulations were validated by pressure drop measurements and the numerical DSD by measurements obtained using dynamic light scattering. The deviation for the Sauter mean diameter was again demonstrated only for one experiment and was found to be $7.4 \%$.

A critical look on the reported literature related to the use of PBE in static mixers points out the lack of significant experimental validation of the developed methodologies. Moreover, in the aforementioned studies, the focus was only on the effect of the superficial velocity on the final DSD while other operating conditions are important and should be investigated to totally validate the robustness of the proposed approaches. In this study, a PBE model is developed for the prediction of the evolution of the DSD in SMX+ static mixers when only breakage occurs. The model is validated against a large range of experimental data obtained in different conditions. The effects of the number of SMX+ elements, the viscosity and the fraction of the dispersed phase, the volume-average energy dissipation rate as well as the DSD at the inlet of the mixers are investigated. 


\section{Materials and experimental methods}

\subsection{Emulsion ingredients}

Oil-in-water emulsions are prepared by dispersing silicon oil (Bluestar Silicone, France) in deionized water which constitutes the continuous phase. Polysorbate 20 (Tween20 ${ }^{\circledR}$, supplied by Sigma-Aldrich, Germany) is used as surfactant. Four different silicon oils of viscosities 20, 50, 100 and $350 \mathrm{mPa} . \mathrm{s}$ are investigated in this study.

\subsection{Static mixers}

SMX+ static mixers (supplied by Sulzer) are used for the emulsification process. SMX+ is a stainless steel made mixer composed of six bars in each direction inclined by $45^{\circ}$ to the tube axis. Each SMX+ mixer has its length equal to its diameter $(5 \mathrm{~mm})$ as shown in Figure (1). As announced by the manufacturer, the SMX + static mixer achieves the same mixing performance as the standard SMX Sulzer but at lower pressure drop due to its less dense structure [40].
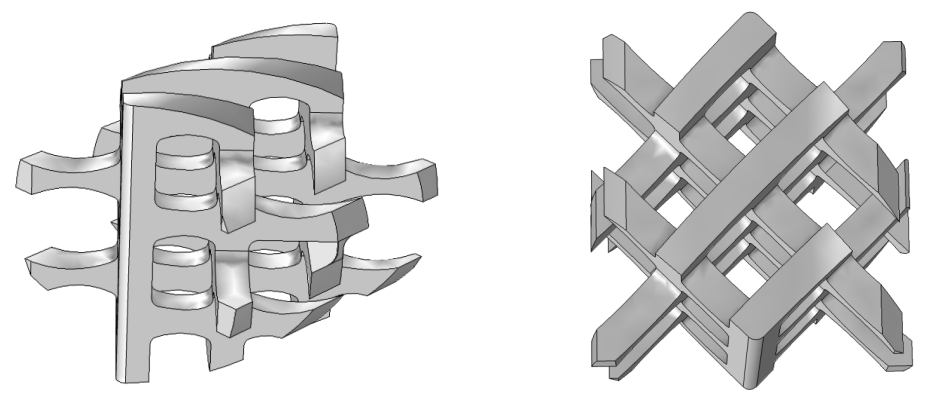

Figure 1: 3D geometry of the SMX+ static mixer used in this study. The CAO was kindly provided by Sulzer.

\subsection{Experimental setup and emulsification procedure}

The experimental set-up used in this study is schematically shown in Figure (2). A pre-emulsion is first prepared by mixing the emulsion ingredients in a 1-L mechanically-stirred tank. The reactor is equipped with 4 vertical blade baffles, and an impeller consisting of six-blade two-disk Rushton turbine. The blades of the impeller are welded to two parallel disks with the upper one containing a hole. Therefore, besides the creation 
of radial flow, this specific impeller design creates a pumping effect and enhances the reactor uniformity. A constant rotation speed of $500 \mathrm{rpm}$ is used in all experiments. Under these conditions, 2 hours of stirring are needed to reach a mean droplet diameter of around $100 \mu m$ (when the viscosity of the dispersed phase is 50 mPa.s). The prepared pre-emulsions were found to be stable in time for several hours, which allowed using ex-situ techniques for the DSD measurement.

Once the pre-emulsion is prepared, it is pumped through the static mixers using a MCP-Z Ismatec ${ }^{\circledR}$ gear pump equipped with Micropump ${ }^{\circledR}$ magnetically driven heads. A pressure gauge (0-6 bar, \pm 3 mbar $)$ is installed after the gear pump to measure the differential pressure. Two samples of each experiment are withdrawn before and after the static mixers tube, to measure the droplet size distribution using a laser diffraction device (Mastersizer 3000, Malvern Instruments, UK). The Mastersizer is based on laser diffraction and gives a volume size distribution. All measurements were realized three times and the mean value was then considered.

The SMX + static mixers are inserted into a transparent polyvinyl chloride tube of same diameter (5 mm) in order to ensure a good adhesion and avoid radial rotations. When more than one static mixer is used, each additional SMX + is put in contact with the previous one and rotated by an angle of $90^{\circ}$ to minimize preferential fluid flow pathways.

The number of SMX+ static mixers is varied between 1 and 10 elements. Four different silicon oils of viscosities 20, 50, 100 and $350 \mathrm{mPa}$.s are used with a volume fraction ranging between 1 to $5 \%$ are investigated. The effect of volume-average energy dissipation rate is explored by varying the fluid superficial velocity, which is done by manipulating the flow rate of the gear pump. Finally, to vary the DSD at the inlet of the mixers, the premixing time in the stirred reactor is varied. These different conditions are summarized in Table 1 . 


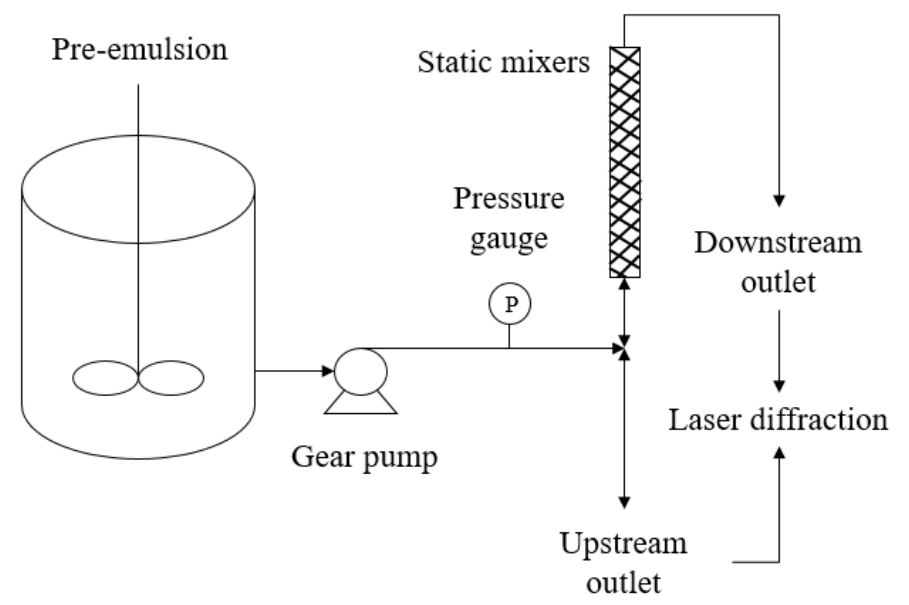

Figure 2: Schematic representation of the experimental set-up

\begin{tabular}{|c|c|c|c|c|c|}
\hline Experiment & Number of SMX + & $\mu(\mathrm{mPa} . \mathrm{s})$ & $\varepsilon\left(\mathrm{m}^{2} / \mathrm{s}^{3}\right)$ & $\phi(\%)$ & Premixing time $(\mathrm{h})$ \\
\hline 1 & 1 & 50 & 314 & 1 & 2 \\
2 & 4 & 50 & 314 & 1 & 2 \\
3 & 8 & 50 & 314 & 1 & 2 \\
4 & 10 & 50 & 314 & 1 & 2 \\
\hline 5 & 10 & 20 & 314 & 1 & 2 \\
6 & 10 & 100 & 314 & 1 & 2 \\
7 & 10 & 350 & 314 & 1 & 2 \\
\hline 8 & 10 & 50 & 172 & 1 & 2 \\
9 & 10 & 50 & 430 & 1 & 2 \\
10 & 10 & 50 & 626 & 1 & 2 \\
\hline 11 & 10 & 50 & 314 & 2 & 2 \\
12 & 10 & 50 & 314 & 3 & 2 \\
13 & 10 & 50 & 314 & 4 & 2 \\
14 & 10 & 50 & 314 & 5 & 2 \\
\hline 15 & 10 & 50 & 314 & 1 & 4 \\
\hline
\end{tabular}

Table 1: Summery of the experimental conditions investigating: the effect of the number of SMX+ inserted in the pipe; the oil viscosity; the volume-average energy dissipation rate; the volume fraction of the dispersed phase and the premixing time (i.e. the DSD at the inlet of the mixers).

\section{Theoretical framework}

\subsection{Modeling droplet breakage in $S M X+$ static mixers}

Due to the low volume fraction of the dispersed phase which does not exceed $5 \%$ in all cases on one hand and the presence of a high concentration of surfactant on the other hand, coalescence is neglected 9 , 20]. 
Moreover, silicon oil has a negligible solubility in water which leads to insignificant mass transfer between the two phases and avoids Ostwald ripening (droplets growth). Thus only droplet breakage is considered in this study. The population balance equation governing the time evolution of the DSD where only breakage occurs in a spatially homogeneous system (the convection in and out of the control volume and the slip velocity between the dispersed and continuous phases are neglected) is given by the following equation:

$$
\frac{\partial n(v, t)}{\partial t}=\int_{v}^{\infty} \beta\left(v, v^{\prime}\right) \Gamma\left(v^{\prime}\right) n\left(v^{\prime}, t\right) d v^{\prime}-\Gamma(v) n(v, t)
$$

where $n(v, t)$ is the number based DSD density, $v$ the droplet volume, $\Gamma(v)$ the breakage frequency for a droplet of size $v$ and $\beta\left(v, v^{\prime}\right)$ is the daughter size distribution function giving the distribution of daughter droplets generated by the breakage of a droplet of volume $v^{\prime}$. The first term on the right hand side of equation (1) accounts for the birth of droplets of volume $v$ resulting from the breakage of bigger droplets $v^{\prime}$ and the last term is the death term due to the loss of droplets of volume $v$ because of their breakage.

\subsubsection{Breakage frequency}

Narsimhan et al. 41] proposed a model for the transitional breakage probability of droplets in agitated liquid-liquid dispersions. They stated that the mechanism of breakage of droplets is due to their oscillations as they are exposed to a turbulent field. Droplets are then expected to break if the turbulent motion provides

the minimum increase in the surface energy for breakage. The velocity fluctuations are induced by the arriving turbulent eddies of different scales. By assuming that the eddy-droplet collisions are statistically regular events forming a Poisson process and the viscous forces are negligible within drops compared to surface forces, they derived the following breakage rate model:

$$
\Gamma(v)=C_{1} \operatorname{erfc}\left(\sqrt{\frac{\sigma}{\rho_{c}}} \frac{C_{2}}{\varepsilon^{1 / 3} v^{5 / 18}}\right)
$$


where $C_{1}$ and $C_{2}$ are constants, $\sigma$ the surface tension of the dispersed phase, $\rho_{c}$ the density of the continuous phase and $\varepsilon$ is the energy dissipation rate. Erfc denotes the complementary error function.

Alopaeus et al. 11 followed a similar approach as Narsimhan et al. 41, but they further included the viscous forces effect on the droplet breakage process. The breakage frequency is expressed in its final form as :

$$
\Gamma(v)=C_{1} \varepsilon^{1 / 3} \operatorname{erfc}\left(\sqrt{C_{2} \frac{\sigma}{\rho_{c} \varepsilon^{2 / 3} v^{5 / 9}}+C_{3} \frac{\mu_{d}}{\sqrt{\rho_{c} \rho_{d}} \varepsilon^{1 / 3} v^{4 / 9}}}\right)
$$

where $\mu_{d}$ and $\rho_{d}$ are respectively the viscosity and the density of the dispersed phase.

The turbulence damping effect on turbulent energy dissipation is adopted from Alopaeus et al. [42] as:

$$
\varepsilon=\varepsilon_{c}\left(\frac{(1-\phi)\left(\phi \rho_{d} / \rho_{c}+(1-\phi)\right)}{1+1.5 \phi \frac{\mu_{d}}{\mu_{d}+\mu_{c}}}\right)^{3}
$$

where $\phi$ is the volume fraction of the dispersed phase and $\mu_{c}$ the viscosity of the continuous phase.

The use of this kernel was preferred in this work as it is based on fundamental investigations, it accounts for the main properties of the dispersion (i.e. viscosities, fraction, densities, energy dissipation) and it is quite simple to implement.

\subsubsection{Daughter size distribution function}

For a complete description of droplets breakage, in addition to the breakage frequency, the daughter size distribution function is needed. It gives the size of the daughter droplets formed after a single fragmentation event. As for the breakage kernels, different daughter size distribution functions were proposed in literature (see the review by Liao \& Lucas [19]). A statistical model is used in this study by assuming that the size of a daughter droplet is a random variable with its probability distribution given by the beta distribution below 
[19]:

$$
\beta\left(v, v^{\prime}\right)=30 \frac{m}{v^{\prime}}\left(\frac{v}{v^{\prime}}\right)^{2}\left(1-\left(\frac{v}{v^{\prime}}\right)\right)^{2}
$$

where $m$ is the number of droplets formed after a breakage event.

Binary breakage is assumed to take place and in this case, one should note that the daughter distribution function satisfies the equations of conservation and symmetry :

$$
\left\{\begin{aligned}
\int_{0}^{v^{\prime}} \beta\left(v, v^{\prime}\right) d v & =m=2 \\
\beta\left(v, v^{\prime}\right) & =\beta\left(v^{\prime}-v, v^{\prime}\right)
\end{aligned}\right.
$$

\subsection{Volume-average energy dissipation rate in SMX+ static mixers}

Emulsification systems are conducted under turbulent conditions, where droplet breakage is governed by turbulent energy dissipation. In static mixers, the volume-average energy dissipation rate is related to the pressure drop and the velocity of the fluid by the following expression:

$$
\varepsilon=\frac{\Delta P u_{s}}{\rho_{c} L \varphi}
$$

where $\Delta P$ is the pressure drop, $L$ the length of the static mixer elements, $\varphi$ is the global porosity of the SMX + which is equal to $75 \%$ and $u_{s}$ the superficial velocity of the fluid (empty tube) given by the following equation:

$$
u_{s}=\frac{4 Q}{\pi D^{2}}
$$

where $Q$ is the volume flow rate of the fluid and $D$ the diameter of the tube.

In the current study, it is assumed that the energy dissipation rate inside the static mixers is uniform, without spatial distribution. Thus, the volume-average energy dissipation rate is used to implement the 
PBE-based model. Note that equation (7) gives the energy dissipation of the dispersion (i.e. $\varepsilon$ ). Thus, it takes into account the oil fraction, the viscosities and the densities of both phases. The way how these parameters are related to the energy dissipation of the continuous phase is shown by equation (4).

\subsection{Numerical solution of the PBE}

To solve the breakage PBE numerically, the finite volume method is adopted [43]. In this method [43], the continuous space is discretized into a certain number of cells $\Lambda_{i}=\left[x_{i-1 / 2}, x_{i+1 / 2}\right]$. The finite volume scheme is based on the conservation of the total mass of the system achieved through the mass fluxes exchanged between the individual cells of the distribution. From the standard population balance equation accounting for breakage (1), the equivalent mass conservation law is derived as :

$$
\frac{\partial g(x, t)}{\partial t}=\frac{\partial x n(x, t)}{\partial t}=\frac{\partial}{\partial x}\left(\int_{x}^{\infty} \int_{0}^{x} v \beta\left(v, v^{\prime}\right) \Gamma\left(v^{\prime}\right) n\left(v^{\prime}, t\right) d v d v^{\prime}\right)
$$

For a cell $i$ at time $t$, the mass flux accross the cell boundaries $\left(x_{i-1 / 2}, x_{i+1 / 2}\right)$ is given by:

$$
\frac{d n_{i}}{d t}=\frac{J_{i+1 / 2}-J_{i-1 / 2}}{x_{i+1 / 2}-x_{i-1 / 2}}
$$

with the mass flux :

$$
J_{i+1 / 2} \approx-\sum_{j=i+1}^{I} g_{j} \int_{\Lambda_{j}} \frac{\Gamma\left(v^{\prime}\right)}{v^{\prime}} d v^{\prime} \int_{0}^{x_{i+1 / 2}} v \beta\left(v, x_{j}\right) d v
$$

A geometric discretization of the space coordinate (droplet volume) is chosen in this study with 100 total grid cells starting from $0.01 \mu \mathrm{m}$. The DSD is measured at the inlet of the SMX + static mixers, after the gear pump as well as at the outlet, after the mixers for each experiment. The inlet DSD is used as an initial value of the DSD in the PBE. The residence time of the emulsion in the system of SMX+ mixers is evaluated to be of the order of few milliseconds (around $10 \mathrm{~ms}$ for the first experiment in Table 1). The model is simulated 
over the residence time of the experiment under investigation. The model output is then compared to the outlet experimental DSD.

\section{Results and discussion}

\subsection{Identification of the model parameters}

As it can be seen in the breakage model, three parameters need to be identified for the present system. Two experiments were chosen in order to identify these parameters: experiments 5 and 7 . These two experiments include a strong variation in dispersed phase viscosity. The used minimization criterion is the difference between the measured and the model-predicted DSD in volume at the outlet of the mixers. The obtained fitting parameters are shown in Table 2

\begin{tabular}{|c|c|c|}
\hline$C_{1}$ & $C_{2}$ & $C_{3}$ \\
\hline 4.06 & 0.59 & $\sim 0$ \\
\hline
\end{tabular}

Table 2: Breakage kernel parameters obtained by optimization using the experiments 5 and 7 of Table 1

These values are different from those found in the literature for stirred tanks when the tank is considered to be uniform (i.e. no coupling with CFD nor compartmentalization). This is due to the differences between the two systems in terms of the spatial uniformity of mixing. Indeed, in the stirred tank, a mean, quite low, energy dissipation is usually computed and used in the kernels, while CFD studies indicate a high energy dissipation in a small region around the impeller and very low energy dissipation elsewhere in the tank. The small region around the impeller should be the one responsible for breakage. Using a mean energy dissipation value is a big approximation. In stirred tanks, it is more appropriate to employ either coupling with CFD, or compartmentalization or the recently proposed averaged kernel based on the distribution of energy dissipation 44. As the static mixers offer a better spatial uniformity of energy dissipation than the stirred tank, it can be believed that the obtained parameters are more representative to the real phenomena taking place in the breakage region. 
Equation (3) shows that the viscous and surface forces are presented as opposing forces to the disruption force due to turbulence. Therefore, $C_{2}$ would reflect the effect of the surface forces compared to disrupting forces on breakup; $C_{3}$ would evaluate the impact of the dispersed phase viscosity compared to disrupting forces; finally $C_{1}$ is proportional to the breakage term and is supposed to be the main parameter reflecting the effect of the energy dissipation on breakup. These parameters were found by Alopaeus et al. [1] to be of the order $C_{1}=0.986, C_{2}=0.892 \times 10^{-3}, C_{3}=0.2$, where a compartmentalization approach was employed. In the present work, the obtained value of $C_{1}$ is higher and that of $C_{2}$ is smaller. However, we think that the static mixer geometry is more adapted to estimate these parameters as the considered volume remains smaller than the considered zone around the impeller in the compartmentalization model. Concerning the parameter $C_{3}$ (estimated here at $2 \cdot 2 \cdot 10^{-14}$ ), it appears that it is not sensitively affecting the estimations, and can be taken off from the model. However, the effect of the viscosity of the dispersed phase is still present in the model as the energy dissipation of the dispersion is affected by the viscosity of the dispersed phase (equation (4) as well as experimentally in equation (7)).

The identified model was then used to predict the evolution of the DSD under different conditions, in the following sections, without new identification.

\subsection{Influence of the number of SMX+ elements}

The number of static mixers can affect the evolution of the DSD until equilibrium is reached between the turbulent droplet disruption/oscillation leading to breakage and entity cohesion due to surface energy phenomena. In this study, the number of mixers was varied as follows $1,4,8$ and 10 . The other parameters were fixed as follows: the viscosity $\mu_{d}=50 \mathrm{mPa.s}$, the chosen flow rate gives an average energy dissipation of $\varepsilon=314 \mathrm{~m}^{2} / \mathrm{s}^{3}$ and the oil fraction $\phi=1 \%$.

The effect of the number of SMX+ elements on the measured evolution of the DSD is reported in Figure (3). It can be seen that increasing the number of mixers leads to a decrease in the droplet size and to a 
narrower distribution. The equilibrium between turbulent surface disruption and surface energy is reached after 8 mixers, and therefore the results obtained with 8 and 10 mixers are similar. Note that this equilibrium depends on the flow rate, oil fraction and physico-chemical properties of both phases. Thus, under different conditions it might be reached with a different number of mixers. It can also be noticed that the distribution is quite large, and definitely it cannot be described by a mean value.

Note that when plotting the size distribution in number it moves towards smaller sizes. Therefore, the $d_{32}$ is calculated to be as small as $30 \mu \mathrm{m}$ while $d_{50}$ by volume is $70 \mu \mathrm{m}$, and none of them is representative of the total distribution.
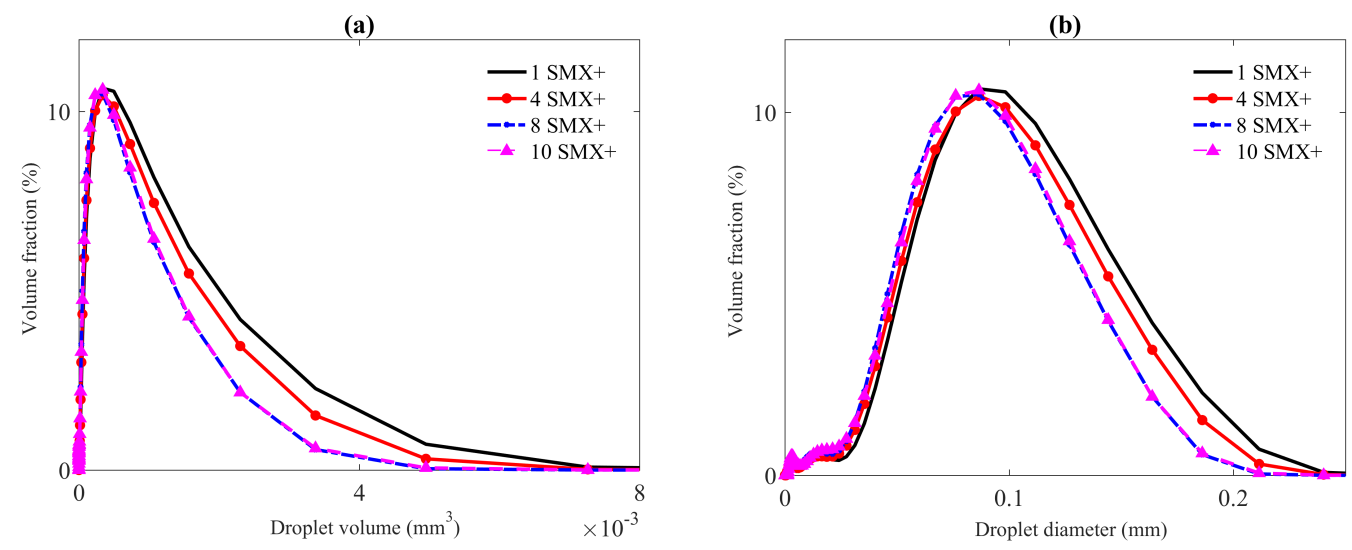

Figure 3: Effect of the number of SMX+ elements on the final DSD: experimental results obtained by laser diffraction (experiments 1, 2, 3 and 4 of Table 1). The x-axis in Figure 3a is plotted in the volume domain and that of Figure 3b in the diameter domain.

Figure (4) shows the modeling results when varying the number of SMX+ elements. A good agreement between the experimental and modeling results can be observed for all numbers of mixer elements. A big gain is thus obtained in this model compared to steady-state correlations which usually do not allow the prediction of the DSD for different numbers of mixers, except few of them which account for the number of mixers in an empirical way. Moreover, the present model allows to have a prediction of the whole size distribution and not only a mean size. 

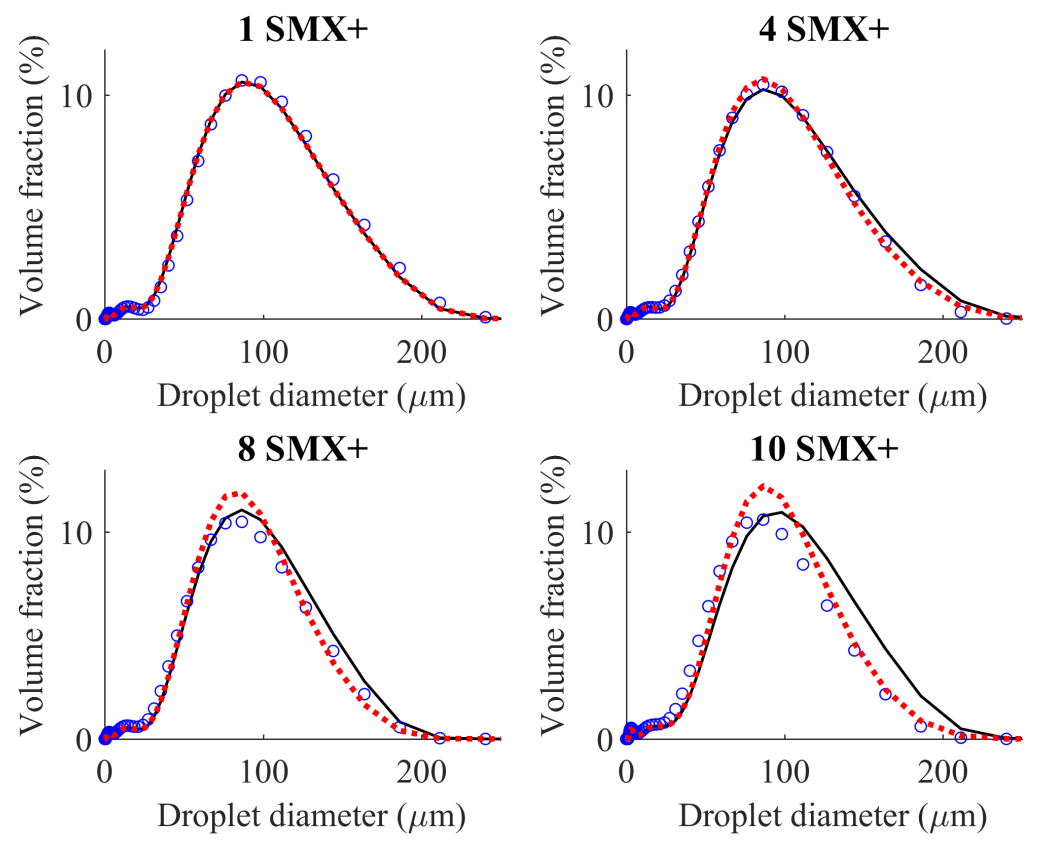

Figure 4: Comparison of the model predictions to experimental results when varying the number of SMX+ elements. The continuous lines are related to the initial experimental DSD at the upstream, the circles are the experimental downstream results and the dashed lines are the PBE based model predictions.

\subsection{Influence of the oil viscosity}

As mentioned earlier, four different silicone oils were considered in this series, with viscosities of $\mu_{d}=$ 20, 50, 100 and $350 \mathrm{mPa} . \mathrm{s}$. The other process parameters were fixed as follows: number of SMX+ mixers $=10, \varepsilon=314 \mathrm{~m}^{2} / \mathrm{s}^{3}$ and oil fraction $\phi=1 \%$. Figure (5) shows the experimental as well as the modeling results of these experiments (at the outlet of the static mixers). It can be seen that increasing the viscosity leads to the formation of bigger droplets. This observation can be explained by the fact that viscous droplets oscillate less than less viscous ones. Thus, the provided turbulent energy has to overcome both the surface energy as well as the viscous energy to cause breakage. It can be seen that the model is able to take these phenomena into account, as the viscosity of the dispersed phase is accounted for in the breakage frequency and in the energy dissipation that considers the damping effect proposed by Alopaeus et al. 11. Note that the models of Narsimhan et al. [41] or Coulaloglou \& Tavlarides 32] would not be able to predict the effect 
of the viscosity of the dispersed phase as they consider only the surface forces. These results demonstrate that the identified parameters are representative of the system as they could predict the evolution of oils with different viscosities that were not included in the identification step. Figure (5) also shows that when increasing the viscosity of the dispersed phase, the DSD tends to become wider. This shape was formed in the reactor (figure not shown) and was conserved after passing in the static mixers.

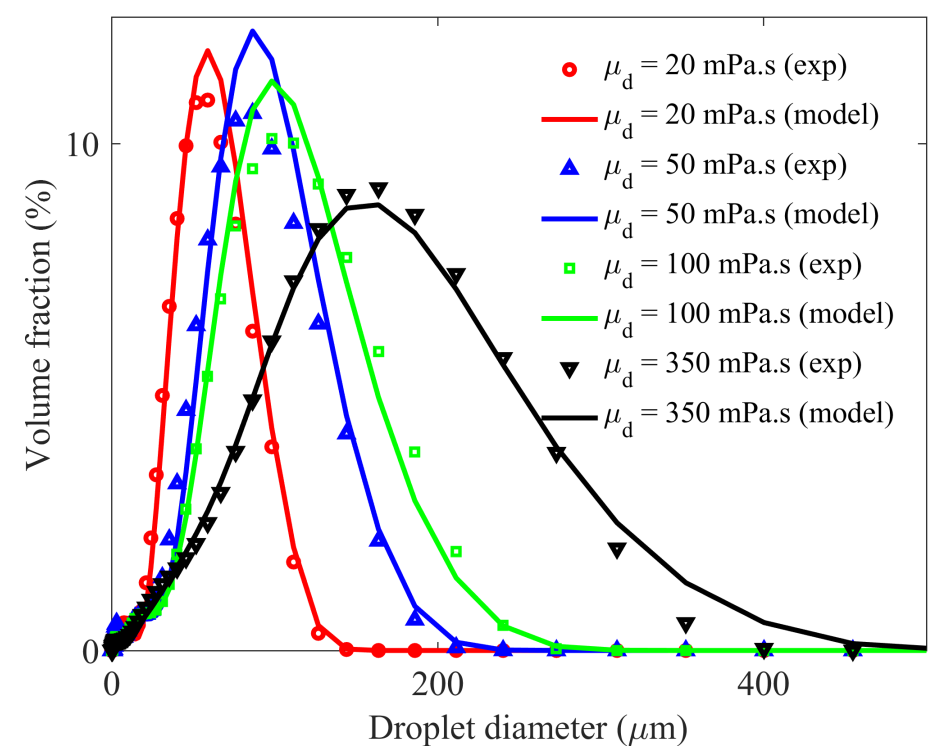

Figure 5: Comparison between the experimental results (symbols) and the model predictions (continuous lines) of the DSD when varying the viscosity of the dispersed phase (experiments $4,5,6$ and 7 of Table 1 .

\subsection{Influence of the oil volume fraction}

The volume fraction of the dispersed phase was varied as follows: $\phi=1,2,3,4$ and $5 \%$. Under these quite dilute conditions, coagulation can still be considered to be negligible. The other parameters were fixed as follows: number of SMX + mixers $=10, \varepsilon=314 \mathrm{~m}^{2} / \mathrm{s}^{3}$ and oil viscosity $\mu_{d}=50 \mathrm{mPa.s}$.

Figure (6) shows the experimental results of this series. It can be seen that, within this range, the oil fraction has a low effect on the evolution of the DSD. However, a general tendency of increasing of the mean size can be observed when increasing the oil fraction. This can be due to the damping effect on energy dissipation. Figure (7) shows the modeling results compared to each experimental result of this series. It can 


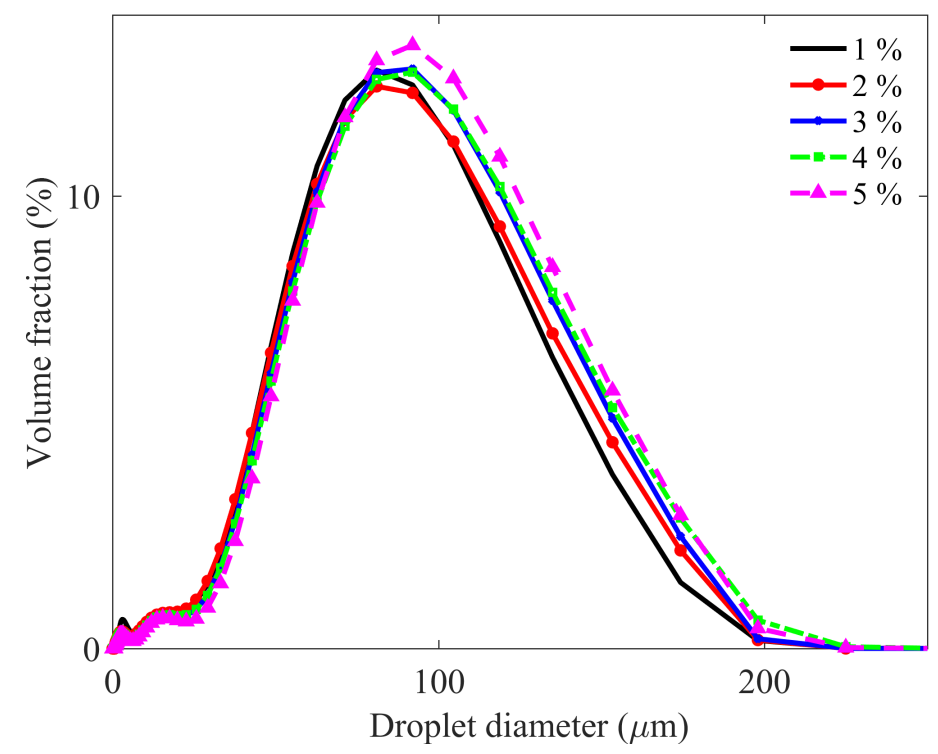

Figure 6: Effect of the volume fraction of the dispersed phase on the final DSD: experimental results (experiments 4, 11, 12, 13 and 14 of the table 1 .

be seen that the model fits the experiments. This confirms that the change in the DSD can be predicted by the damping effect alone and that coalescence is indeed negligible in this system.
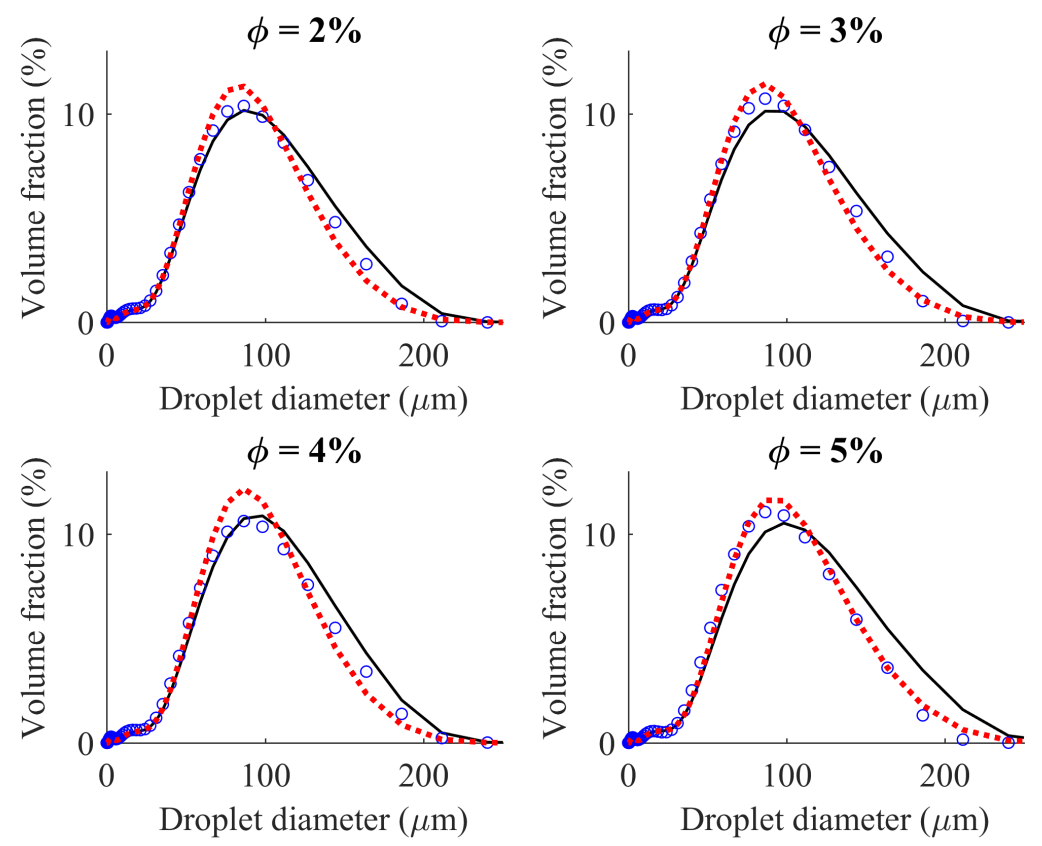

Figure 7: Comparison between the model predictions and the experimental results when varying the dispersed phase volume fraction. The continuous lines are related to the initial experimental DSD at the upstream, the circles are the experimental downstream results and the dashed lines are the PBE based model predictions. 


\subsection{Influence of the volume-average energy dissipation rate}

The flow rate of the pre-emulsion was varied as follows $Q=10.1,11.9,13.4,14.8 \mathrm{~mL} . \mathrm{s}^{-1}$, which led to volume-average energy dissipation values of $\varepsilon=172,314,430,626 \mathrm{~m}^{2} / \mathrm{s}^{3}$ (equations (7) and (8)). Note that the energy dissipation also depends on the number of static mixers that affects pressure drop (equation (7)). The other parameters were fixed as follows: number of SMX + mixers $=10$, oil fraction $\phi=1 \%$ and oil viscosity $\mu_{d}=50 \mathrm{mPa}$.s. Figure $(8)$ shows the experimental results of this series. It can be seen that increasing the energy dissipation leads to a net decrease in the droplet size. Indeed, the breakage mechanism is governed by the turbulence which increases when increasing the flow rate.

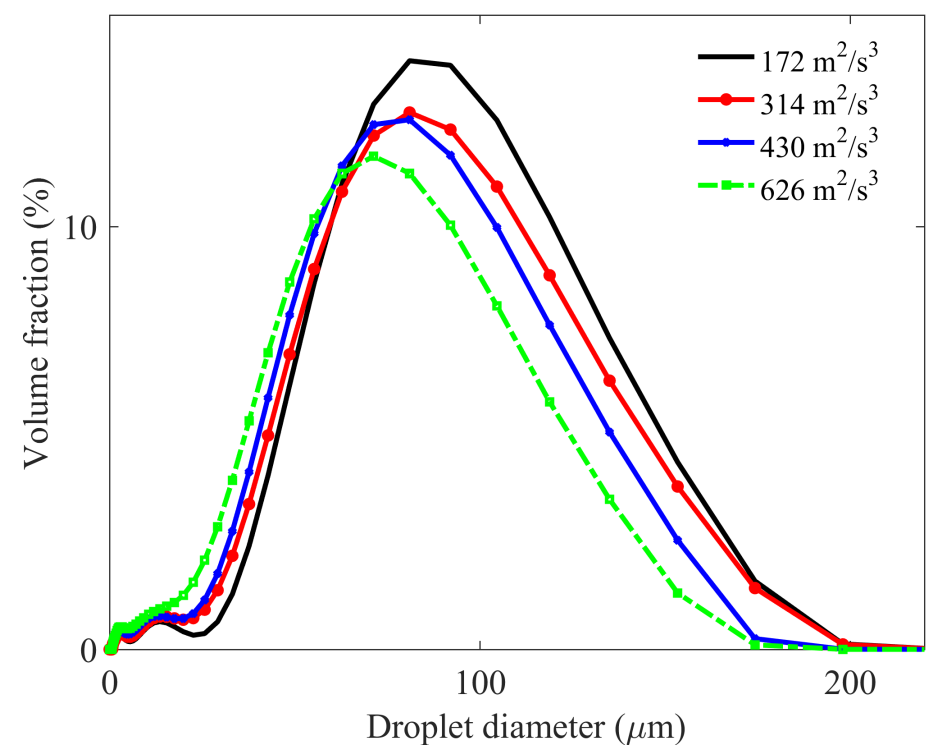

Figure 8: Effect of the volume-average energy dissipation rate on the final DSD: experimental results (experiments $4,8,9$ and 10 of Table 1 .

Figure (9) shows the modeling results compared to the experimental measurements for each experiment.

It can be seen that the model again fits quite well to the experimental data. This validates that the identified model correctly describes the effect of $\varepsilon$, and can be generalized to wide mixing conditions. 

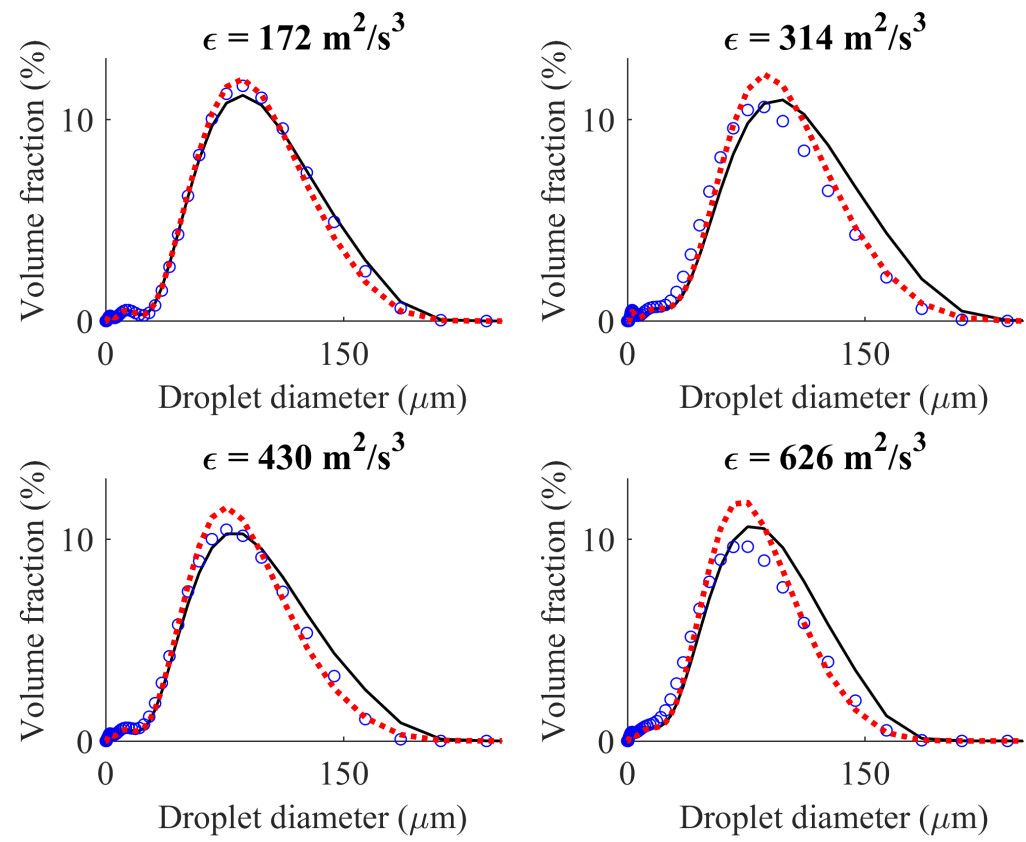

Figure 9: Comparison between the model predictions and the experimental results when varying the volume-average energy dissipation rate. The continuous lines are related to the initial experimental DSD at the upstream, the circles are the experimental downstream results and the dashed lines are the PBE based model predictions.

\subsection{Influence of the DSD at the inlet of the $S M X+$ mixers}

In this series, the size of the DSD at the inlet of the mixers was varied by mixing it in the stirred tank for different durations: 2 and $4 \mathrm{~h}$. The objective behind this series is to figure out wether the droplet size at the outlet of the mixers is independent of the input as usually claimed in steady-state correlations used to represent breakage in static mixers. Indeed, the inlet size is not involved in such correlations and it is assumed that the number of mixers is sufficient to ensure equilibrium between disrupting forces (e.g. turbulence) and cohesion forces (e.g. surface or viscous forces, coalescence). The number of mixers used in this series was 10. The other parameters were fixed as follows: turbulent energy dissipation $\varepsilon=314 \mathrm{~m}^{2} / \mathrm{s}^{3}$, oil fraction $\phi=1$ $\%$ and oil viscosity $\mu_{d}=50$ mPa.s. Note that under these conditions, 8 mixers were found to be enough to reach equilibrium and no further breakage was observed when putting more mixers (Figure (3)).

Figure (10) shows the experimental inlet and outlet DSD values as well as the numerically predicted 
outlets. It can be seen that the DSD obtained at the outlet of the mixers indeed depends on the inlet DSD. It seems that bigger droplets require more mixers to reach equilibrium. Moreover, injecting bigger droplets in the mixers leads to a higher breakage rate inside the mixers while smaller droplets cross the mixers without undergoing much breakage as they are already close to the equilibrium conditions.

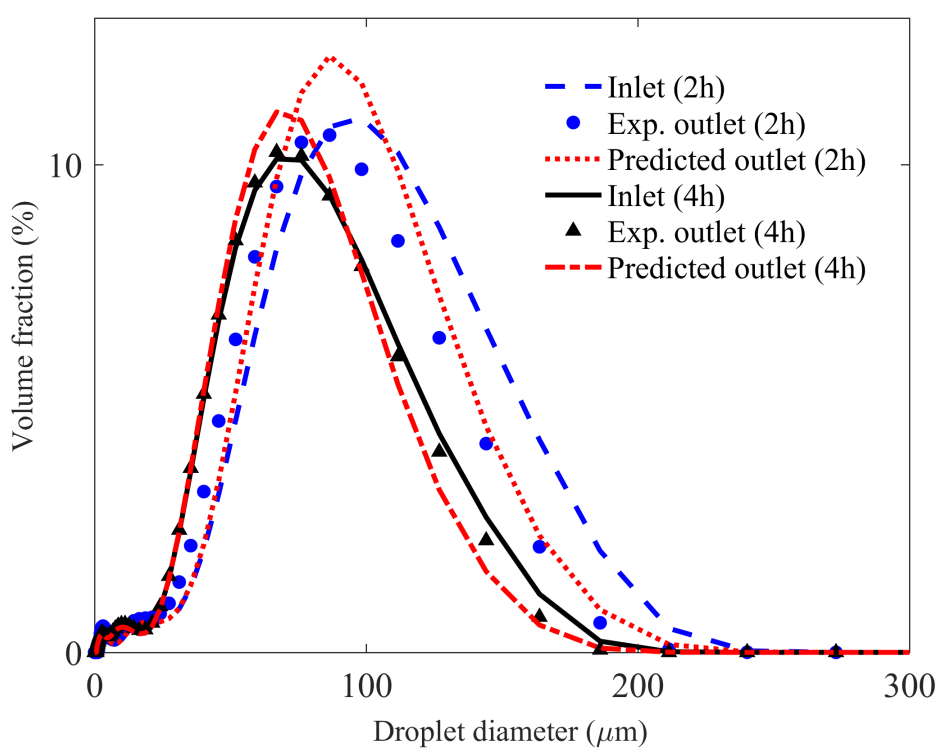

Figure 10: Comparison between the model predictions and the experimental results when varying the DSD at the inlet of the SMX + mixers (experiments 4 and 15 of Table 1 .

\section{Discussion}

The influence of the dispersed phase properties and operating conditions on the rate of droplets breakage were explored first experimentally by measuring the DSD at the inlet and the outlet of the mixer elements by laser diffraction. To do this, a pre-emulsion was prepared in a stirred tank reactor and pumped through the static mixers in the turbulent regime. This experimental procedure allows a precise control of the fraction of the dispersed phase as well as the inlet DSD. The variation of the viscosity of the dispersed phase (from 20 to 350 mPa.s, experiments: $4,5,6,7)$ shows a strong effect on the DSD at the inlet and outlet of the mixers. This indicates that the higher the oil viscosity the lower is the breakage rate. Moreover, the DSD is wider 
when the viscosity increases (Figure (5)).

When varying the dispersed phase fraction from 1 to $5 \%$ (experiments: $4,11,12,13,14$ ), the consequence on the DSD is not very important as the system is dilute enough to keep coalescence negligible. A general tendency can however be observed where the increase of dispersed phase fraction shifts the DSD to bigger sizes (Figure (6)), which means that one needs more energy to reduce the size of concentrated emulsions. This is due to the damping effect on energy dissipation.

In terms of operating conditions, first the number of SMX+ elements was varied from 1 to 10 (experiments: $1,2,3,4)$. As the energy dissipation rate is high in systems involving static mixers, the residence time inside these mixers is of critical importance. When increasing the number of SMX+ elements, the residence time is increased which means that the pre-emulsion faces high energy dissipation for longer times. This explains the shift of the DSD toward small droplets. After 8 SMX + mixers, no breakage is observed as the steady-state equilibrium is reached, under the conditions of this series of experiments. Under the present conditions where coalescence is negligible, the equilibrium indicates equality between disrupting forces (due to turbulence) and cohesion forces (i.e. surface and viscous forces).

The energy dissipation rate inside the static mixers depends on the pressure drop and the fluid velocity. The pressure drop is imposed by the nature and the number of static mixers, while the fluid velocity depends on the flow rate delivered by the pump. A large range of $\varepsilon$ (from 172 to $626 \mathrm{~m}^{2} / \mathrm{s}^{3}$ ) was explored in this study. The results shown in Figure (8) point out a noticeable decrease of the DSD when the energy dissipated is higher which is in concordance with the physics of the turbulent breakage phenomenon. Finally, the DSD of the emulsion at the inlet of the mixers is varied, by varying the pre-emulsion preparation period. When the pre-emulsion preparation in the stirred tank reactor is extended from 2 to 4 hours, the DSD becomes smaller and tends to a similar steady-state as when using 10 SMX+ elements under the considered operating conditions. Therefore, the rate of breakage in Figure 10 is low in the mixers when 4 hours of pre-mixing 
is considered. This further confirms that steady state is highly dependent on the operating conditions. Therefore, it is more reasonable to develop a model that predicts breakage based on the inlet DSD.

At the light of these experimental results, one has to highlight that all of them reflect the physics of liquid-liquid dispersions when breakage prevails. This large amount of valuable data constitutes a database for the modeling part of this study.

When dealing with dispersed systems, the population balance framework has shown its potential in a wide range of applications to predict the evolution of the particle/droplet size distribution. In this work, the population balance equation accounting for breakage is adopted for the modeling of droplets breakage process, as coalescence can be assumed to be negligible under dilute conditions with a high amount of surfactant. This model allows to capture the evolution of the full DSD when classical correlations only give access to a truncated information of the DSD (generally the mean size). As demonstrated in the experimental part, the breakage process depends both on the surface and the viscous forces, and therefore the breakage kernel proposed by Alopaeus et al. [1] is adopted. For the daughter size distribution, a statistical model is used by assuming a binary and random breakage with a beta distribution as its probability function. The proposed PBE-based model has 3 adjustable parameters that were identified by an optimization procedure using only two different experiments. The identified model was then exploited extensively to predict the evolution of the DSD under different conditions. Concerning the computational cost, except for the optimization procedure, all the numerical simulations take less than 1 second under a standard personal computer.

The experimental versus numerical DSD comparisons are shown for the effect of: the number of SMX+ elements (Figure (4)), the dispersed phase viscosity (Figure (5)), the dispersed phase volume fraction (Figure (7)), the volume-average energy dissipation rate (Figure (9)) and the DSD at the inlet of the SMX+ mixers (Figure (10)). In all these cases, the numerical predictions fit the experimental data. This demonstrates the ability of the current model to track the evolution of the DSD as a function of the different parameters of the 
system. As spatial uniformity can almost be assumed to prevail in the mixers regarding energy dissipation, they might be assumed to lead to better predictions of the breakage kernels over stirred tanks especially if no CFD or compartmentalization is considered.

\section{Conclusion}

In this study, oil-in-water emulsification process using SMX+ static mixers in turbulent regime was investigated. The case of diluted systems was considered. In these conditions, only breakage is assumed to occur while coalescence and Ostwald ripening are negligible. A population balance model accounting for breakage was proposed based on the breakage kernel of Alopaeus et al. 1] and a statistical daughter size distribution function. The model has as input the DSD at the inlet of the SMX+ mixers and predicts the DSD at the outlet. After the identification of the model parameters over two different experiments, the model prediction capabilities were tested against a large range of experimental conditions.

The model predictions were found to be in good agreement with the experimental data for all the investigated parameters even when strong variations on the DSD were reported as in the case of viscosity and volume-average energy dissipation rate. The ability of the model to capture accurately the full DSD makes it a valuable tool for studying emulsification using static mixers. It might also be assumed that the identified parameters are more appropriate for the considered dispersed system thanks to their spatial uniformity, while model identification in stirred tank by assuming spacial uniformity is supposed to lead to big approximations. Finally, the proposed model should be able to predict the droplet size evolution of other types of mixers, as the geometry effects are accounted for in the measured pressure drop.

\section{Aknowledgments}

The authors thank Justine Massias for her help in the experimental part of this work. 


\section{Nomenclature}

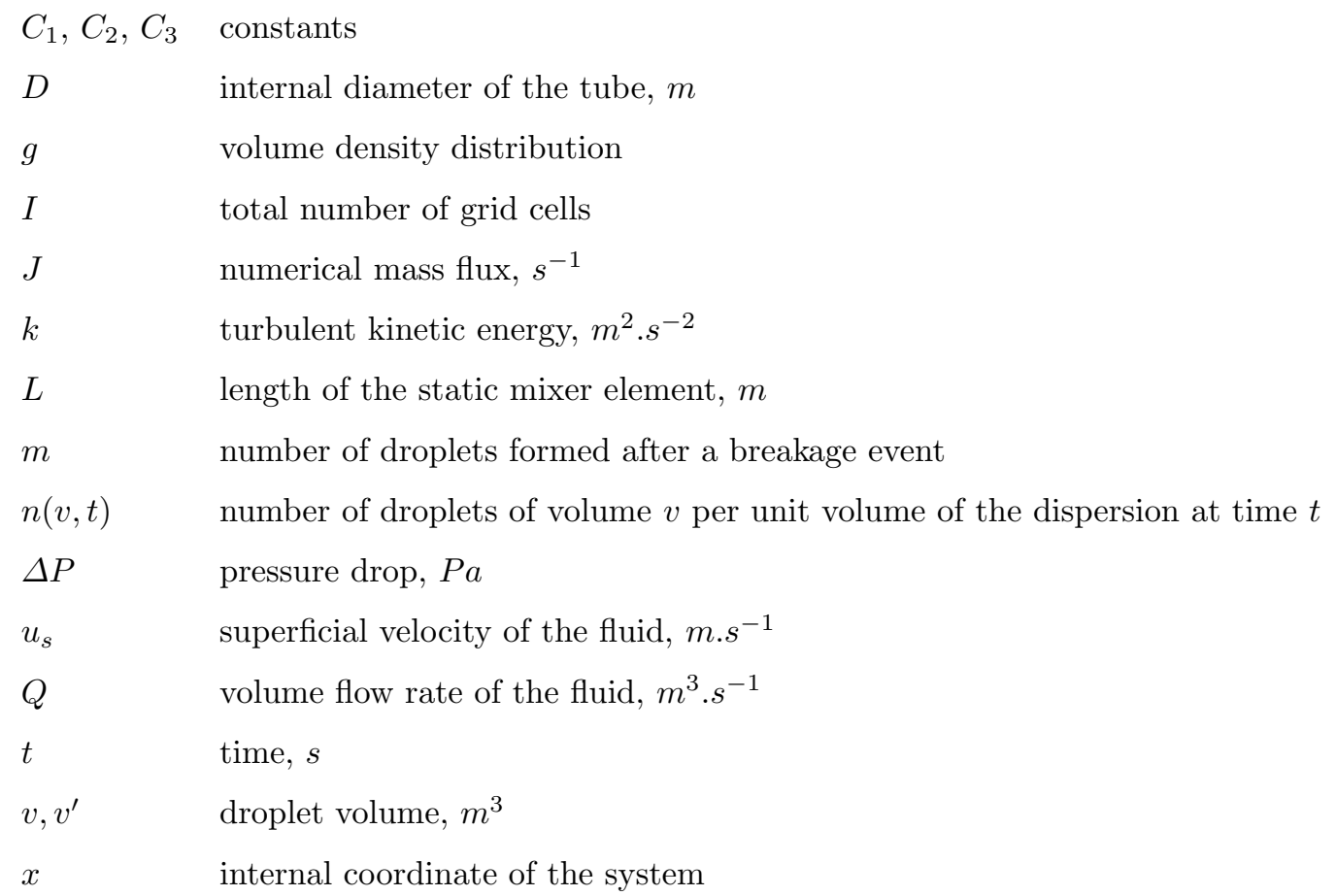

\section{Greek symbols}

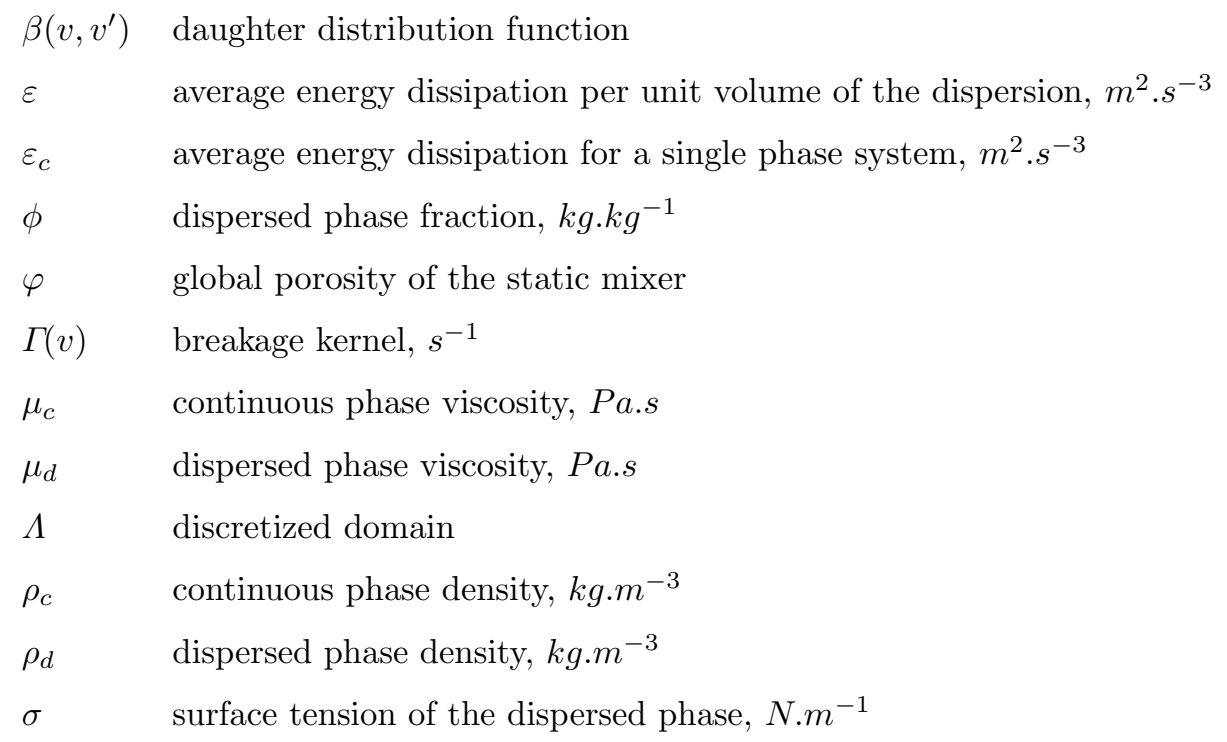

\section{Abbreviations}

CFD computational fluid dynamics

DSD droplet size distribution

PBE population balance equation 


\section{References}

[1] V. Alopaeus, J. Koskinen, K. I. Keskinen, J. Majander, Simulation of the population balances for liquidliquid systems in a nonideal stirred tank. Part 2parameter fitting and the use of the multiblock model for dense dispersions, Chemical Engineering Science 57 (2002) 1815-1825.

[2] S. Marcos, M. Meinecke, A. Kilzer, M. Petermann, Study of LL water-in-oil dispersions generated in SMX-Plus static mixers with dissolved CO 2 under high pressure, The Journal of Supercritical Fluids (2017).

[3] R. Thakur, C. Vial, K. Nigam, E. Nauman, G. Djelveh, Static Mixers in the Process IndustriesA Review, Chemical Engineering Research and Design 81 (2003) 787-826.

[4] M. Coroneo, G. Montante, A. Paglianti, Computational fluid dynamics modeling of corrugated static mixers for turbulent applications, Industrial \& Engineering Chemistry Research 51 (2012) 15986-15996.

[5] A. Ghanem, T. Lemenand, D. Della Valle, H. Peerhossaini, Static mixers: Mechanisms, applications, and characterization methodsA review, Chemical Engineering Research and Design 92 (2014) 205-228.

[6] F. Theron, N. Le Sauze, A. Ricard, Turbulent Liquid- Liquid Dispersion in Sulzer SMX Mixer, Industrial \& Engineering Chemistry Research 49 (2009) 623-632.

[7] H. E. H. Meijer, M. K. Singh, P. D. Anderson, On the performance of static mixers: A quantitative comparison, Progress in Polymer Science 37 (2012) 1333-1349.

[8] P. K. Das, J. Legrand, P. Moranais, G. Carnelle, Drop breakage model in static mixers at low and intermediate Reynolds number, Chemical Engineering Science 60 (2005) 231-238.

[9] N. B. Raikar, S. R. Bhatia, M. F. Malone, M. A. Henson, Experimental studies and population balance 
equation models for breakage prediction of emulsion drop size distributions, Chemical Engineering Science 64 (2009) 2433-2447.

[10] A. M. Al Taweel, C. Li, H. G. Gomaa, P. Yuet, Intensifying mass transfer between immiscible liquids: using screen-type static mixers, Chemical Engineering Research and Design 85 (2007) 760-765.

[11] M. Briceno, J. L. Salager, J. Bertrand, Influence of Dispersed Phase Content and Viscosity on the Mixing of Concentrated Oil-in-Water Emulsions in the Transition Flow Regime, Chemical Engineering Research and Design 79 (2001) 943-948.

[12] S. Middleman, Drop size distributions produced by turbulent pipe flow of immiscible fluids through a static mixer, Industrial \& Engineering Chemistry Process Design and Development 13 (1974) 78-83.

[13] R. V. Calabrese, T. P. K. Chang, P. T. Dang, Drop breakup in turbulent stirred-tank contactors. Part i: Effect of dispersed-phase viscosity, AIChE Journal 32 (1986) 657-666.

[14] J. Legrand, P. Moranais, G. Carnelle, Liquid-Liquid Dispersion in an SMX-Sulzer Static Mixer, Chemical Engineering Research and Design 79 (2001) 949-956.

[15] T. Lemenand, D. Della Valle, Y. Zellouf, H. Peerhossaini, Droplets formation in turbulent mixing of two immiscible fluids in a new type of static mixer, International Journal of Multiphase Flow 29 (2003) 813-840.

[16] M. I. I. Zainal Abidin, A. A. Abdul Raman, M. I. Mohamad Nor, Mean drop size correlations and population balance models for liquidliquid dispersion, AIChE Journal 61 (2015) 1129-1145.

[17] E. Chabanon, N. Sheibat-Othman, O. Mdere, J. P. Valour, S. Urbaniak, F. Puel, Drop size distribution monitoring of oil-in-water emulsions in SMX+ static mixers: Effect of operating and geometrical conditions, International Journal of Multiphase Flow 92 (2017) 61-69. 
[18] S. Maass, A. Gabler, A. Zaccone, A. R. Paschedag, M. Kraume, Experimental Investigations and Modelling of Breakage Phenomena in Stirred Liquid/Liquid Systems, Chemical Engineering Research and Design 85 (2007) 703-709.

[19] Y. Liao, D. Lucas, A literature review of theoretical models for drop and bubble breakup in turbulent dispersions, Chemical Engineering Science 64 (2009) 3389-3406.

[20] S. Maass, N. Paul, M. Kraume, Influence of the dispersed phase fraction on experimental and predicted drop size distributions in breakage dominated stirred systems, Chemical Engineering Science 76 (2012) 140-153.

[21] L. Han, S. Gong, Y. Li, Q. Ai, H. Luo, Z. Liu, Y. Liu, A novel theoretical model of breakage rate and daughter size distribution for droplet in turbulent flows, Chemical Engineering Science 102 (2013) $186-199$.

[22] W. Wang, W. Cheng, J. Duan, J. Gong, B. Hu, P. Angeli, Effect of dispersed holdup on drop size distribution in oilwater dispersions: Experimental observations and population balance modeling, Chemical Engineering Science 105 (2014) 22-31.

[23] D. Li, Z. Gao, A. Buffo, W. Podgorska, D. L. Marchisio, Droplet breakage and coalescence in liquidliquid dispersions: Comparison of different kernels with EQMOM and QMOM, AIChE Journal 63 (2017) 22932311.

[24] A. Hakansson, C. Tragardh, B. Bergenstahl, Dynamic simulation of emulsion formation in a high pressure homogenizer, Chemical Engineering Science 64 (2009) 2915-2925.

[25] N. B. Raikar, S. R. Bhatia, M. F. Malone, D. J. McClements, C. Almeida-Rivera, P. Bongers, M. A. Henson, Prediction of emulsion drop size distributions with population balance equation models of 
multiple drop breakage, Colloids and Surfaces A: Physicochemical and Engineering Aspects 361 (2010) $96-108$.

[26] S. N. Maindarkar, N. B. Raikar, P. Bongers, M. A. Henson, Incorporating emulsion drop coalescence into population balance equation models of high pressure homogenization, Colloids and Surfaces A: Physicochemical and Engineering Aspects 396 (2012) 63-73.

[27] A. Hakansson, F. Innings, C. Tragardh, B. Bergenstahl, A high-pressure homogenization emulsification modelImproved emulsifier transport and hydrodynamic coupling, Chemical Engineering Science 91 (2013) 44-53.

[28] A. Dubbelboer, J. Janssen, H. Hoogland, A. Mudaliar, S. Maindarkar, E. Zondervan, J. Meuldijk, Population balances combined with Computational Fluid Dynamics: A modeling approach for dispersive mixing in a high pressure homogenizer, Chemical Engineering Science 117 (2014) 376-388.

[29] P. J. Becker, F. Puel, A. Dubbelboer, J. Janssen, N. Sheibat-Othman, Coupled population balanceCFD simulation of droplet breakup in a high pressure homogenizer, Computers \& Chemical Engineering 68 (2014) 140-150.

[30] Z. Gao, D. Li, A. Buffo, W. Podgrska, D. L. Marchisio, Simulation of droplet breakage in turbulent liquidliquid dispersions with CFD-PBM: Comparison of breakage kernels, Chemical Engineering Science 142 (2016) 277-288.

[31] F. Azizi, A. M. A. Taweel, Turbulently flowing liquidliquid dispersions. Part I: Drop breakage and coalescence, Chemical Engineering Journal 166 (2011) 715-725.

[32] C. A. Coulaloglou, L. L. Tavlarides, Description of interaction processes in agitated liquid-liquid dispersions, Chemical Engineering Science 32 (1977) 1289-1297. 
[33] F. Azizi, A. M. A. Taweel, Hydrodynamics of Liquid Flow Through Screens and Screen-Type Static Mixers, Chemical Engineering Communications 198 (2011) 726-742.

[34] Z. Jaworski, P. Pianko-Oprych, D. L. Marchisio, A. W. Nienow, CFD Modelling of Turbulent Drop Breakage in a Kenics Static Mixer and Comparison with Experimental Data, Chemical Engineering Research and Design 85 (2007) 753-759.

[35] H. Luo, H. F. Svendsen, Theoretical model for drop and bubble breakup in turbulent dispersions, AIChE Journal 42 (1996) 1225-1233.

[36] P. D. Berkman, R. V. Calabrese, Dispersion of viscous liquids by turbulent flow in a static mixer, AIChE Journal 34 (1988) 602-609.

[37] E. Bayraktar, O. Mierka, F. Platte, D. Kuzmin, S. Turek, Numerical aspects and implementation of population balance equations coupled with turbulent fluid dynamics, Computers \& Chemical Engineering 35 (2011) 2204-2217.

[38] G. A. Farzi, N. Rezazadeh, A. Parsian Nejad, Homogenization Efficiency of Two Immiscible Fluids in Static Mixer Using Droplet Tracking Technique, Journal of Dispersion Science and Technology 37 (2016) 1486-1493.

[39] J. Solsvik, H. A. Jakobsen, On the solution of the population balance equation for bubbly flows using the high-order least squares method: implementation issues, Reviews in Chemical Engineering 29 (2013) $63-98$.

[40] S. Hirschberg, R. Koubek, F.Moser, J. Schck, An improvement of the Sulzer SMX static mixer significantly reducing the pressure drop, Chemical Engineering Research and Design 87 (2009) 524-532. 
[41] G. Narsimhan, J. P. Gupta, D. Ramkrishna, A model for transitional breakage probability of droplets in agitated lean liquid-liquid dispersions, Chemical Engineering Science 34 (1979) 257-265.

[42] V. Alopaeus, J. Koskinen, K. I. Keskinen, Simulation of the population balances for liquidliquid systems in a nonideal stirred tank. Part 1 Description and qualitative validation of the model, Chemical Engineering Science 54 (1999) 5887-5899.

[43] J. Kumar, G. Warnecke, M. Peglow, S. Heinrich, Comparison of numerical methods for solving population balance equations incorporating aggregation and breakage, Powder Technology 189 (2009) 218-229.

[44] A. Buffo, J. De Bona, M. Vanni, D. L. Marchisio, Simplified volume-averaged models for liquidliquid dispersions: correct derivation and comparison with other approaches, Chemical Engineering Science 153 (2016) 382-393. 